\title{
Opinion paper: is there a role for breeding for welfare improvement?
}

\author{
S. P. Turner ${ }^{\dagger}$, J. Conington and C. M. Dwyer \\ Animal and Veterinary Sciences Research Group, SRUC, Kings Buildings, Edinburgh, EH9 3JG, UK
}

(Received 16 December 2014; Accepted 31 March 2015; First published online 24 April 2015)

\section{Introduction}

Increasing global demand for animal products produced with ever greater efficiency makes it unlikely that the pressure placed on livestock industries and therefore the animals themselves will diminish in the foreseeable future. Increasing affluence and awareness of welfare issues by society may drive improvements in welfare standards, but this may be regional rather than global in impact. Some complex welfare problems in intensive production systems, such as tail biting in pigs and feather pecking in hens, have existed for decades, have significant negative impacts on economic and environmental sustainability but have known solutions that are too costly for many producers to implement. Other welfare challenges, such as poor health control or high neonatal mortality in extensively managed systems, persist because management options for their mitigation are limited. Still other welfare challenges have been exacerbated in the past by imbalanced selective breeding on a narrow range of economically important traits, most notably in the dairy and broiler industries. Considerable variation exists between animals in their expression of negative welfare outcomes (e.g. in aggressive behaviour in pigs; Figure 1). Selective breeding leads to permanent and cumulative change, and breeding for appropriately targeted traits has the potential to benefit welfare without negative economic impacts or the requirement for major management change. This article will focus on three examples of welfare problems that have persisted for many decades and are tolerated as routine within current production systems, but which have the potential for improvement via selection. These examples are diverse and present ethical dilemmas, each to a different extent, with regard to the acceptability of using selection to improve welfare. The first example (improving lamb survival) is the least controversial of the three examples. The second (improving sheep resistance to foot infections known as 'footrot') is likely to benefit welfare but major improvements ought to be achievable on some farms without recourse to breeding through better prophylactic and therapeutic health management. The final example

\footnotetext{
${ }^{\dagger}$ E-mail: simon.turner@sruc.ac.uk
}

(reducing pig aggression) deliberately seeks to reduce expression of behaviours that are an integral part of the repertoire shown by all pig breeds and wild boar since housing systems that minimise this problem are not economically feasible.

\section{Lamb survival}

Worldwide pre-weaning lamb mortality averages $15 \%$ to $20 \%$ with nearly $50 \%$ of lamb deaths occurring on the day of birth. Death through starvation and hypothermia is likely to be prolonged (Dwyer, 2008). This represents a major welfare, economic and environmental burden in an industry that is a cornerstone of regional economies. Despite considerable research, the mortality rate has not improved in recent decades and management options to protect or treat neonatal lambs are typically limited in extensive production systems. Neonatal lamb vigour and ewe maternal behaviour have been shown to be major determinants of survival. Lamb vigour is easy to record, has a higher genetic correlation with survival than ewe maternal behaviour and is a moderately heritable trait with high genetic variance (e.g. reduced ability to suck; heritability 0.32, SE 0.04; Matheson et al., 2012). Furthermore, it is not genetically correlated with lamb growth. The heritability compares favourably with that of traits currently under selection in the global sheep industry (e.g. fecundity; heritability 0.16 ) meaning that it ought to be technically possible to selectively breed for improved lamb survival. Simple scores for lamb vigour, sucking ability and lambing ease have been developed and are measurable by farmers, and are able to accurately estimate genetic propensity for survival. Uptake of new innovations by the sheep industry is constrained by labour availability and the poorly integrated nature of the industry. However, developing scores for lamb vigour in partnership with farmers has resulted in their use now beginning on commercial farms.

\section{Footrot}

Lameness caused by virulent footrot strains of the anaerobic bacteria Dichelobacter nodosus is a debilitating, painful 
Turner, Conington and Dwyer

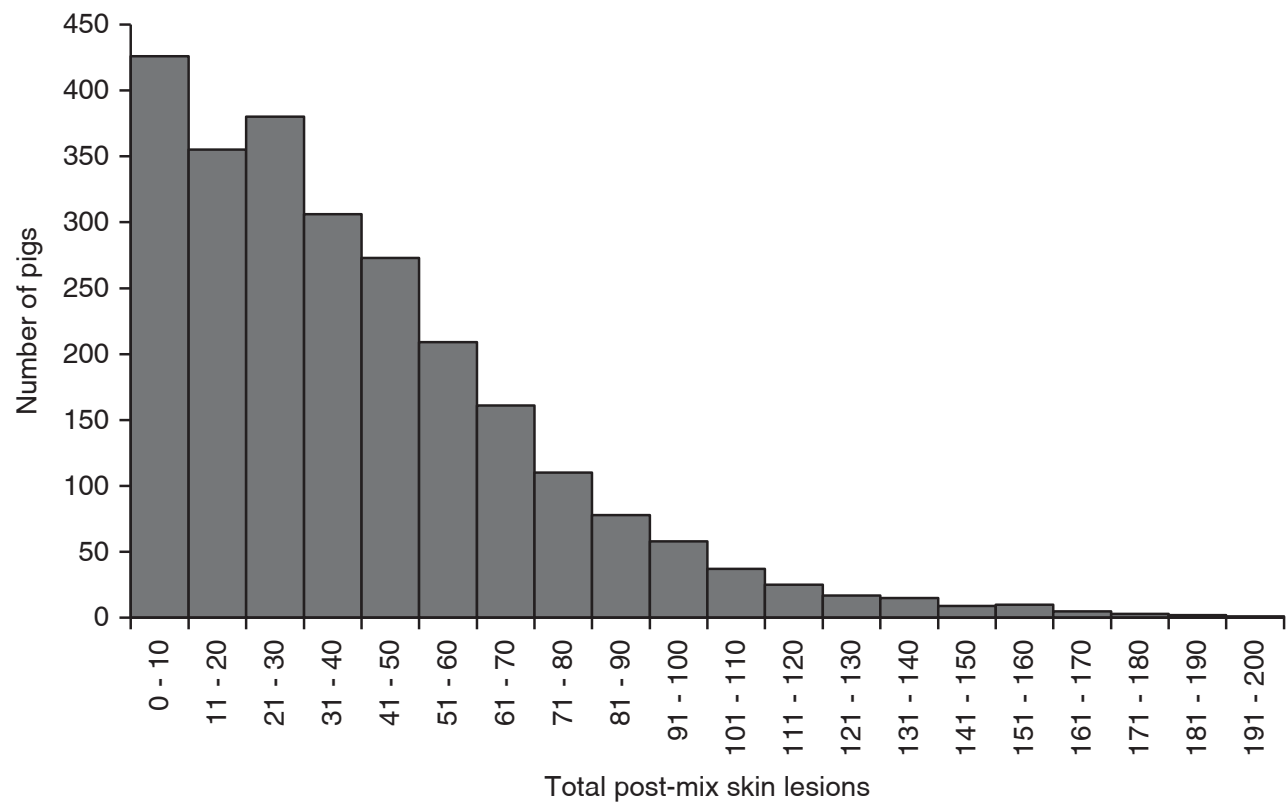

Figure 1 Frequency distribution of the number of fresh skin lesions present on pigs $24 \mathrm{~h}$ after regrouping into new social groups. The number of lesions has been genetically associated with involvement in reciprocal and non-reciprocal aggressive behaviour (Turner et al., 2009).

hoof infection. Footrot is endemic in the major sheepproducing countries with wetter climates such as the UK and Ireland, with prevalence levels of footrot hoof lesions of between $13 \%$ and $23 \%$ and actual lameness of between $8 \%$ and $10 \%$. Infection can be prevented and controlled by careful hygiene for housed ewes, foot-bathing, promptly treating affected ewes and separating them from the main flock until they are cured. There is undoubtedly a role for better awareness of its diagnosis, prevention and control strategies, but efficient management can be problematic even on wellmanaged farms. Footrot resistance is heritable (heritability 0.15 to 0.25 ; Raadsma and Dhungyel, 2013) and is associated favourably with number of lambs reared and unfavourably with lamb liveweight gain. Simple 5-point scoring methods exist and are being used in some countries to provide phenotypic data on affected hooves for use in sheep breeding programmes to reduce footrot prevalence (Raadsma and Conington, 2010).

\section{Pig aggression}

The majority of commercially farmed pigs experience regrouping into new social groups at least once in order to house animals together of similar weight and to ensure available buildings are used to maximum capacity. Fighting to establish new dominance relationships can be intense and can lead to many skin lesions. The quality of behaviour performed is similar to that performed between wild boar, but the quantity of aggression is typically greatly increased in commercial production due to the sudden grouping of animals of similar competitive ability and in close proximity in an environment that prevents escape. Low-cost, labour efficient methods to reduce aggression have minimal benefits and the avoidance of regrouping is economically unviable for many producers. Large variation exists in aggressiveness
(Figure 1) and some aggressive behavioural traits have heritabilities only slightly below that of growth rate (e.g. duration in reciprocal fighting; heritability 0.43 SE 0.04 ; Turner et al., 2009). Recording of skin lesion number and location can provide a rapid estimation of the genetic propensity of pigs to engage in damaging reciprocal fighting (e.g. genetic correlation with count of lesions to the front third of the body 0.67 , SE 0.04; Turner et al., 2009). However, recording lesion numbers for all selection candidates remains a barrier to implementation. Current effort to understand the genomic architecture of aggressiveness may provide the means to avoid routine phenotyping and facilitate implementation of selection.

\section{Future perspective and conclusion}

Modern breeding tools will increase the accuracy of selection while facilitating the improvement of traits that are difficult or costly to routinely record. With this, the feasibility is being enhanced of using selection alongside improved management to make positive progress in addressing some of the most intractable welfare problems. The remaining barriers to implementation vary by industry and trait, but share common themes. Better estimation and communication of the total economic and non-economic costs associated with specific welfare conditions is required to motivate change. Phenotyping costs, even in the genomic era, will remain prohibitive unless rapid but sensitive indicator traits are developed similar to those given in the examples above. Lastly, the correlated consequences for economic and other welfare-relevant traits needs to be assessed for most of the welfare traits that may be targeted for improvement. These barriers need to be addressed in partnership with the breeding industry and farmers. To realise benefits in welfare, selection of animals that are more able to thrive in commercial production systems must not 
simply allow management to deteriorate such that the net outcome for the animals remains unchanged. With modern breeding techniques comes the attendant risk of accelerating unwanted change and a need to fully assess the correlated consequences for the animals. To return to the example of pig aggression, we have shown that reduced aggressiveness is not associated with activity levels or a tendency to lose fights, but are yet to probe the affective state of pigs that do not engage in fights. It is plausible that these animals do not feel the motivation to fight, are better able to avoid unnecessary fights or are afraid of fighting, each of which have different implications for their likely affective state. Whether selection will be successful and justifiable will probably have to be assessed on a case by case basis. Examples exist in which progress has been made in simultaneously benefitting animal welfare and economic productivity through broader breeding goals, for example through the Profitable Lifetime Index in the UK dairy industry that places selection pressure on resistance to mastitis and lameness in addition to production traits. The decision on whether to target welfare traits through breeding is likely to be easier for some cases (e.g. neonatal survival) than others (e.g. complex social behaviours) and to be acceptable to different degrees by retailers and consumers. However, where economically feasible management solutions and legislation alone have proved incapable of improving long-standing, routine and serious welfare issues, selective breeding may have a future role alongside continued efforts to find effective management solutions.

\section{References}

Dwyer CM 2008. The welfare of the neonatal lamb. Small Ruminant Research 76, 31-41.

Matheson SM, Bünger L and Dwyer CM 2012. Genetic parameters for fitness and neonatal behavior traits in sheep. Behavior Genetics 42, 899-911.

Raadsma HW and Conington J 2010. Genetic aspects of resistance to ovine footrot. In Breeding for disease resistance in farm animals; 3rd edition (ed. SC Bishop, RFE Axford, FW Nicholas and JB Owen), pp. 251-276. CABI Publishing, Wallingford, UK.

Raadsma HW and Dhungyel OP 2013. A review of footrot in sheep: new approaches for control of virulent footrot. Livestock Science 156, 115-125.

Turner SP, Roehe R, D'Eath RB, Ison SH, Farish M, Jack MC, Lundeheim N, Rydhmer L and Lawrence AB 2009. Genetic validation of post-mixing skin injuries in pigs as an indicator of aggressiveness and the relationship with injuries under more stable social conditions. Journal of Animal Science 87, 3076-3082. 